The following curious example illustrates this fact.

Suppose we have a regular heptagon, a regular hexagon, a regular pentagon and four equilateral triangles, all the sides being of equal length, and we wish to make a polyhedron from them, each solid angle being a trihedral angle.

The number of faces $F=7$; the number of edges

$$
E=\frac{1}{2}(7+6+5+4.3)=\frac{1}{2} \quad 30=15 \text {, }
$$

the number of vertices $V=\frac{1}{3} .30=10$.

Hence $F+V=17=E+2$, and Euler's condition is fulfilled.

But it is obviously impossible to construct such a surface, as there are only 6 other faces to fit to the 7 sides of the heptagon.

1074. Note on approximations.

H. V. Mallison.

This note provides an alternative to a section of Mr Inman's article, "What is Wrong with the Teaching of Approximations?" (Gazette, XVI, December 1932, p. 306).

In the case of products, say $(A \pm h)(B \pm k)$, I suggest the following straightforward method.

$\begin{array}{cc}\text { Maximum limit } & A B+A k+B h+h k \\ \text { Minimum limit } & \frac{A B-A k-B h+h k}{2 A k+2 B h} \\ \text { Difference } & \end{array}$

If all the measurements were precisely accurate the true product would be

$$
A B \pm h k \pm A k \pm B h .
$$

Now as $h$ and $k$ are fractional, $h k$ is less than either $h$ or $k$, so $h k$ must be omitted, and we are left with $A B \pm A k \pm B h$.

If $h=k$, the product is $A B \pm h(A+B)$.

In example (1), p. $309, A=2 \cdot 68$ and $B=4 \cdot 12$,

$$
h=\cdot 005 \text {, and so } h(A+B)=\cdot 005(6 \cdot 8)=\cdot 034 \text {. }
$$

As only two places of decimals are here allowable, the correct answer is $11 \cdot 04 \pm .03 \mathrm{sq}$ in.

Subtraction requires a little thought. Take for example

$$
\begin{aligned}
& 41 \cdot 3 \pm \cdot 05 \\
& \frac{11 \cdot 2 \pm \cdot 05}{30 \cdot 1 \pm \cdot 1}
\end{aligned}
$$

If the variations are taken of the same sign, the result will be $30 \cdot 1$, but if of contrary sign the result will be either 30 or $30 \cdot 2$.

A. S. Percival.

\title{
ERNEST WILLIAM HOBSON
}

October 27, 1856 - April 18, 1933 